\title{
New Technologies for Kidney Surgery Planning 3D, Impression, Augmented Reality 3D, Reconstruction: Current Realities and Expectations
}

\author{
Francesco Esperto ${ }^{1}$ • Francesco Prata ${ }^{1}$ (D) - Ana María Autrán-Gómez ${ }^{2} \cdot$ Juan Gomez Rivas $^{3} \cdot$ Moises Socarras $^{4}$. \\ Michele Marchioni ${ }^{5} \cdot$ Simone Albisinni ${ }^{6} \cdot$ Rita Cataldo $^{7} \cdot$ Roberto Mario Scarpa $^{1} \cdot$ Rocco Papalia $^{1}$
}

Accepted: 22 April 2021 / Published online: 25 May 2021

(C) The Author(s), under exclusive licence to Springer Science+Business Media, LLC, part of Springer Nature 2021

\begin{abstract}
Purpose of review We aim to summarize the current state of art about 3D applications in urology focusing on kidney surgeries. In addition we aim to provide a snapshot about future perspective of intraoperative applications of augmented reality (AR).

Recent findings A variety of applications in different fields have been proposed. Many applications concern current realities and 3D reconstruction, while some others are about future perspective. The majority of recent studies have focused their attention on preoperative surgical planning, patient education, surgical training, and AR.

Summary The disposability of 3D models in healthcare scenarios might improve surgical outcomes, learning curves of novice surgeons and residents, as well as patients' understanding and compliance, allowing a more shared surgical decision-making.
\end{abstract}

Keywords Three dimensional (3D) $\cdot$ Kidney $\cdot$ Augmented reality $\cdot$ Surgery $\cdot$ Urology

\section{Introduction}

Three-dimensional printing (3DP) has emerged in the late 1980s, but its application in medical field dates to 2000s [1]. Until recently, its use was limited mostly to orthopedics and

This article is part of the Topical Collection on Kidney Diseases

Francesco Prata

francesco.prata@gmail.com

1 Department of Urology, Campus Bio-Medico University, Rome, Italy

2 Department of Urology, Hospital Universitario Fundación Jiménez Diaz, Madrid, Spain

3 Department of Urology, Hospital Clinico San Carlos, Madrid, Spain

4 Department of Urology, Instituto de Cirugia Urologica Avanzada (ICUA), Madrid, Spain

5 Unit of Urology, Department of Medical, Oral and Biotechnological Sciences, SS. Annunziata Hospital, G. D’Annunzio University, Chieti, Italy

6 Urology Department, University Clinics of Brussels, Erasme Hospital, Université Libre de Bruxelles, Brussels, Belgium

7 Unit of Anesthesia, Intensive Care and Pain Management, Department of Medicine, Campus Bio-Medico, University of Rome, Rome, Italy dentistry. As three-dimensional (3D) printers have become more widespread and affordable, a rapid increase in the use of 3DP in medicine has been registered. Currently, several types of technologies are available for printing such as binder jetting, material jetting, vat photopolymerization technologies, and powder bed fusion; furthermore $3 \mathrm{D}$ printers can now generate object from different materials such as plastics, wax, ceramics, and metal [2]. In recent years, 3DP-technology (3DPT) allowed to manufacture models to produce facsimiles of patients' organs, even entire body parts, to be used for training purposes and to improve surgical planning. Moreover, the introduction of artificial realities created with the help of software (virtual realities VR, augmented realities) represents a further step in 3DPT allowing the surgeon to perform guided-surgery without giving up concentration on the operating field.

Aim of this review is to examine the application of 3DP in kidney surgery; in particular we focus on surgical planning, patient education, training, and augmented reality (AR).

\section{DP and Surgical Planning}

Pre-operative planning is crucial to improve surgical outcome and to reduce possible intra- or post-operative complications. $3 \mathrm{D}$ models (3DM) could play an important role helping the 
surgeons to properly understand patient specific anatomy and guiding their intraoperative decisions. 3D anatomical replicas printed directly from patients DICOM images (CT scan or MRI) have been used to aid information in the preoperative planning of complex surgical procedures and trying to reduce perioperative complications [3]. Surgical planning with 3DM has significant advantages over the current two-dimensional (2D) images. Surgeons have a more realistic overview and better comprehension of the area on which they're going to perform surgery. Possible benefits provided from these models are better decision-making and consequently increased surgical confidence.

\section{Applications of 3DP in Nephron Sparing Surgery (NSS)}

More than a half of publications about 3DM in urology reported surgical planning as their primary outcome. In the era of nephron-sparing surgery (NSS), due to the complex anatomy and vasculature, the potential prolonged renal ischemia, and the often unclear tumor depth invasion, is not surprising that the majority of studies have focused their attention on kidney cancer [3].

Even if ablative techniques and active surveillance are possible choices in selected patients, surgical treatment for renal masses still represents the gold standard and has evolved, through years, to a NSS approach. Until 2011, there were no studies proving better outcomes of partial nephrectomy (PN) instead radical nephrectomy (RN) [4]. In 2012, Sun and colleagues demonstrated for the first time that, in multivariable analysis, patients who underwent PN were significantly less likely to die for other-causes mortality (OCM) compared with the RN cohort ( $p=0.04)$, stating that PN should be offered "whenever technically feasible" [5]. Nevertheless, feasible does not always mean easy: higher R.E.N.A.L. score masses are more prone to result in higher Clavien-Dindo postoperative complications $(p=0.043)$ and significant drop in post-operative renal function ( $p=0.004)$, due to surgical complexity [6]. Prior to consider surgical outcomes, preoperative planning can strongly be influenced by $3 \mathrm{DM}$, especially in case of complex renal masses.

Urology has moved from open to minimally invasive surgery (MIS) and from radical organ extirpation to NSS. As a result, surgeons need to rely on models that can give them a better understanding of patients' disease helping in improving surgical planning. Silberstein et al. in 2014 performed 5 PNs (4 robotic and 1 open) with complete excision of renal masses after creating 5 customized, patient-specific, 3D kidney models [7]. The main characteristic of these models was the enhanced renal lesions suspicious for malignancy. In this study, all interventions were successfully performed with an average ischemia time of $21 \mathrm{~min}$, all surgical margins were negative, and complications were minimal. A preliminary report by Zhang et al. showed that, in patients with $\mathrm{T} 1$ renal masses eligible for NSS, 3DMs had higher scores in surgical planning [8]. Furthermore, the two surgeons involved stated that intraoperative consultation of the 3DM was helpful for relationship of the tumor with surrounding tissues, depth of resection, and avoidance of key structures injuries such as renal hilum. However, no specific questions regarding how the 3DMs impacted surgical planning decisions were administered.

After 1 year, Wake et al. selected 10 renal neoplasms scoring more than 5 (range 6-10) at R.E.N.A.L. nephrometry score, and submitted 2D images before 3DMs to three experienced urologic oncology surgeons [9]. After submission of models, $30-50 \%$ of surgeons were prone to change the surgical approach, with the largest impact about transperitoneal or retroperitoneal approach and clamping.

Porpiglia et al. evaluated the usefulness of 3D printed kidney models and surgeon's perception. Based on their data, 3DMs seemed to influence surgeons in the choice of the most appropriate type of ischemia (off-clamp vs global ischemia vs partial ischemia) and the type of resection to perform (enucleation or enucleoresection) without difference on the basis of surgeons' experience [10].

However, good planning doesn't always mean good surgical outcomes. Many studies have focused their attention also on results after 3DMs visualization and to what extent they can influence surgeries. In a feasibility study by Rundstedt et al., a patient-specific presurgical protocol for robotassisted PN (RAPN) was developed [11]. In their study, 10 patients with solid renal masses underwent RAPN after preoperative rehearsal using 3D-printed kidney models made by a silicon-based material. R.E.N.A.L. nephrometry scores were between 7 and 11 (mean 8.9). Authors compared resection times of the model and the tumor. Their results showed no statistically significant difference between the 3DM and the excised tumor in mean resection time (6:58 vs 8:22 min, $p=$ 0.162 ) and volumes excised ( 38.50 vs $41.79 \mathrm{~mm}^{3}, p=0.976$ ). Only $1 / 10$ patients had positive surgical margins. Authors concluded that pre-surgical rehearsal could significantly improve resection strategy, but this study lacks of a control group. Another feasibility study investigating how 3D printed kidney models can influence surgical procedures was performed by Maddox et al. [12]. With the aim of allowing preoperative and robotic surgical simulation, authors constructed patient-specific physical 3DMs made by materials that could approximate quite well the properties of renal tissue. Seven models were successfully created, and PN and renorrhaphy were performed on each replica. After simulation, all patients underwent RAPN with negative surgical margins, reporting an average warm ischemia time of $25 \mathrm{~min}$. Mean R.E.N.A.L. nephrometry score was 7.4. Comparing the seven cases and the "Tulane Urology prospectively maintained RAPN database," surgeries with 3DMs had larger tumors, fewer complications, longer warm ischemic time, fewer positive margins, 
and shorter hospitalization, but the only statistically significant finding was a decreased estimated blood loss (EBL) ( 185.7 vs. $235.6 \mathrm{ml}, p=0.01$ ), suggesting that preoperative 3DM rehearsal may decrease the learning curve for trainees and improve surgical outcomes. Otherwise, authors admitted that further evaluation was needed.

Kyung et al. compared 17 patients who underwent PN aided by prior consultation of 3D patient-specific kidney models with a control group consisting of patients who underwent PN from the same surgeon and approximately during the same time period (2014-2015) [13]. Similarly to Maddox, authors found that the only statistically significant difference was diminished intra-operative blood loss (IBL) (182 cc vs. $310 \mathrm{cc}, p<0.01$ ). No complications occurred in the $3 \mathrm{D}$ printed group. Moreover, patients reported an overall improved understanding of the disease, surgical procedure, and trust in surgical team after consultation of the 3DM.

Despite the relatively small cohort, the frequent lack of a control group and the low statistical power, these studies have contributed to support the efficacy of 3DM simulation before complex renal surgery.

Recently, Fan et al. retrospectively analyzed data of 69 patients who underwent 3D laparoscopic PN (LPN) and 58 who underwent traditional LPN between January 2016 and February 2018 [14]. They reported a significant reduction in warm ischemia time (WIT) in the $3 \mathrm{D}$ group $(24.1 \pm 5.1$ for the $3 \mathrm{D}$ cohort and $26.6 \pm 4.2 \mathrm{~min}$ in the traditional LPN, $p<0.05)$, against a longer surgery waiting time $(13.6 \pm 3.4$ days and 7.0 \pm 0.6 days, respectively, $p<0.05)$. Subgroup analysis according to R.E.N.A.L. scores confirmed that, in patients with scores $\geq 8$, the 3D group had significantly shorter WIT and less IBL $(131.9 \pm 78.5$ vs $179.2 \pm 76.1, p<0.05)$ than the traditional LPN group.

Finally, a Japanese group reported their preliminary experience of what they called "4D surgical navigation" in minimally invasive off-clamp PN [15]. It consists of a full-scale size 3D printed kidney designed so that the tumor and its margin (2-5 $\mathrm{mm}$ around the tumor) could be removed. This feature allowed the surgeon to visualize, during surgical planning, both pre- and post-resection kidney (the fourth considered dimension was the time). Models helped the surgeons to create a working mental map for resection. Ten patients with complex renal masses (R.E.N.A.L. score $\geq 8$ ) were selected and underwent minimally invasive off-clamp PN with acceptable perioperative outcomes and surgical margins that resulted to be nearly identical to $3 \mathrm{D}$ printed tumors. A statistically significant difference was found in the time of intraoperative ultrasound with a $3 \mathrm{D}$ model compared to standard surgeries (mean $3.3 \mathrm{~min}$ vs $6.3 \mathrm{~min}$, respectively, $p=0.021$ ). Moreover, surgeons claimed the usefulness of 3DM tactile feedback. In contrast, 4 to $9 \mathrm{~h}$ were needed for printing and 3 to 9 days to complete the model. Costs of the models ranged from 450 to 680 dollars.

\section{Applications of 3DP in Percutaneous Nephrolithotomy (PCNL)}

Nephrolithiasis is a common disease, and recent data show a prevalence up to $15 \%$ and an overall incidence rate growing [16]. Among endourologic techniques, percutaneous nephrolithotomy (PCNL) represents the gold standard for renal stones larger than $20 \mathrm{~mm}$ due to advantages in operative time and morbidity rates [17]. Renal access isn't always performed by urologists due to a not so easy learning curve. In fact, many studies have focused on imaging modalities used for guidance during percutaneous access for both urologists and radiologists. Anyhow, optimal selection of calyx for puncture and nephrostomy tract is one of the most important steps of PCNL surgical planning. Difficulties include performing a trajectory that leads directly to the target stone without affecting neighbors' structures. Inadvertent organ injuries as well as multiple tracts before achieving the correct calyx access can lead to an increase in surgery duration, higher complications rates, and consequently to a longer post-operative length of stay (LOS) [18]. In recent years, the rapid development of patient-specific 3D segmentation and reconstruction in Urology has led to the combination of patient-specific 3DMs with preoperative planning for needle guidance during PCNL procedures. In 2015, Gadzhiev et al. proposed a plasticine 3D replica of pelvicalyceal system on 32 patients with staghorn stones taken to the operating room and used as a reference model [19]. Percutaneous renal access was performed successfully in all cases; more than a half $(56 \%)$ had a single tract with an overall stone-free rate (SFR) after second look of $87.3 \%$.

In 2019, Xu et al. printed 36 patient-specific 3DMs ( 3 for each patient) and simulated 3 puncture sites from upper-, middle- and lower-pole calyces [20]. The puncture site that achieved the better SFR in the model was then used as reference during surgery, and a good correspondence was founded between post-operative stone volume of the model and of the patient $(p<0.001,95 \% \mathrm{CI})$. In late 2019, an Italian group presented a clinical case of a 30-year-old man with left renal stone $(25 \times 15 \mathrm{~mm})$ [21]. A 3D digital and physical renal model to aid the surgeon during procedure in planning and guiding the percutaneous access during PCNL was performed. The patient safely underwent PCNL with 1 single percutaneous puncture (time of puncture $2 \mathrm{~min}$ ). Overall surgical time was $90 \mathrm{~min}$. Post-operative CT scan confirmed patient's SFR. Brehmer et al. evaluated how 3D-CT reconstructions could influence the choice of access route and treatment outcomes in 35 patients planned for PCNL (88\% with complex renal stone) [22]. Access route were planned on 3DCT reconstructions using anatomical landmarks (ribs, spinous process, etc.). The results were a change of access plan in 15/ 28 patients, while in 7 patients, access could not be planned without 3D-CT, totaling 22/35 (63\%). Sixty-nine percent of these patients $(24 / 35)$ were stone-free after single PCNL. 
Similarly, Li et al. performed image segmentation and 3D reconstruction from $\mathrm{CT}$ scans of 15 patients with complex renal stone, including one patient with a horseshoe kidney, 8 patients with partial/complete staghorn stones, and 6 patients with multiple renal stones [23]. Virtual safe and reliable percutaneous renal access route were established for each patient by comprehensive planning based on the 3DM of renal stones. The safest and most effective percutaneous tract for stone clearance was planned on the models and then uploaded onto a screen during procedure in the operating room. The surgeon could visualize the reconstructed 3DM adjusting kidney transparency and felt more confident and comfortable with the access aided by the virtual puncture. During lithotripsy, the 3D models offered a panoramic view of the stone and collecting system guiding intraoperative nephroscopy. PCNL were completed successfully in all 15 patients, the one-session SFR was $93.3 \%$, and the final SFR was $100 \%$.

Future studies are required, with larger cohorts, but Manning hypothesis of "practice before you play" could become the standard of care for complex surgeries [24].

\section{Patient Education and 3DP}

In the last decade, shared decision-making is becoming more and more widespread since patients claim an increasing role in medical decision-making. In this perspective, pre-operative imaging plays a crucial role in patient counselling and shared surgical decision-making for patients eligible to major kidney surgery [25]. However, many patients experience difficulties in the interpretation of conventional radiological images. To date, there are few 3D guided surgery studies that focused on preoperative patients' education. Assuring to patients an improved understanding of their anatomy and conditions, as well as planned procedures, is often underestimated while it could give a more informed consent and reduce pre-operative anxiety. Many studies have explored possible ways to reduce the comprehension gap between surgeon and patient creating 3DMs and comparing to $2 \mathrm{D}$ imaging in patient undergoing PN. Wake and colleagues prospectively enrolled 49 patients eligible for PN who underwent routine clinical imaging before surgery [26]. The cohort was randomized in two groups: one receiving pre-operative planning with standard imaging and the other with the addition of printed patient-specific 3DM. During surgical planning, a 5-point Likert scale questionnaire was administered to patients and used to determine their understanding. Their study showed better results and consequently a better understanding in the 3DMs cohort compared to $2 \mathrm{D}$ imaging group, with a statistically significant difference in comprehension of cancer size and location $(p=0.04$ and $p=$ 0.012 , respectively), disease and treatment plan $(p=0.014)$, helping the patient to decide consciously to undergo PN instead of RN.
Similar results were showed by Teishima et al. in 29 patients who were candidates to RAPN in 2018 [27]. The 3DM created consisted of the kidney, tumor, ureter, vasculature, and also inferior vena cava and abdominal aorta. A dedicated visual analog scale was used to evaluate perception and understanding. In all patients' questionnaire issues ( $p=0.0006$ in anatomy-related issue, $p=0.0004$ in tumor-related issue, and $p=0.0015$ in procedure-related issue) and in $2 / 3$ of the issues of questionnaire administered to 19 family members $(p=$ 0.0186 in anatomy-related issue and $p=0.0051$ in tumorrelated issue), the $3 \mathrm{DM}$ reached a statistically significantly higher score than conventional CT alone. Moreover, in all $\mathrm{CT}$ issues, 64-year-old or younger patients scored better than the elder ones.

Robot-assisted surgery is not so widespread and affordable, so many centers perform NSS with the aid of laparoscopy. Zhang et al. investigated the impact of 3DMs in T1 renal cancer patients who underwent LPN [28]. From CT images, $103 \mathrm{D}$ kidney models were printed successfully. Renal arteries and veins, collecting system including the ureter, and tumor were preserved and all colored differently, while perirenal fat tissue was removed. Two questionnaires with open ended were plotted. Against a relatively low production price (150 dollars per model), high score were registered among patients (9 or over in all four questions) while, among experienced urologists, details of renal vasculature and the collecting system were scored less favorably.

In 2015, a prospective pilot study was conducted on 7 patients with a primary diagnosis of kidney cancer eligible for PN [29]. From four-phase multi-detector computerized tomography (MDCT) scanning, renal volume data were extracted and a life-size specific 3DM for each patient was printed with transparent resin for renal parenchyma, to better show renal vasculature, collecting system and the renal tumor. Before and after 3DM presentation, questionnaires were administered to patients and their answers analyzed: understanding on kidney physiology $(16.7 \%, p=0.018)$, anatomy $(50 \%$, $p=0.026)$, and planned surgical procedure $(44.6 \%, p=0.026)$ was statistically significant, with an overall improvement of $37.6 \%$.

During an international urological meeting organized on January 2017, Porpiglia and colleagues presented 3DMs of 10 patients who underwent live minimally invasive PN and evaluated the results of 3DMs in overall understanding during a preoperative counseling between patient and surgeon [10]. All patients completed a specifically built Face\&Content questionnaire that showed favorable scores (at least 9/10) about the use of the technology during preoperative case discussion, improving their comprehension of the disease and the intervention.

During the same year, Atalay et al. investigated the feasibility and the impact of personalized 3D-printed pelvicalyceal system models prior to PCNL [30]. Five anatomically 
accurate models of the renal collecting system of patients with unilateral complex renal stones were successfully generated. Authors stated that, after the 3DM presentation, the mean understanding improvement rate was higher, in particular basic kidney anatomy improved by $60 \%(p=0.017)$, kidney stone position by $50 \%(p=0.02)$, planned surgical procedure by $60 \%(p=0.017)$, and understanding of complicationsrelated surgery by $64 \%(p=0.015)$.

Schmit et al., in their pilot prospective study, compared 25 patients of the standard group with as many of the experimental group which received education using a 3D printed renal cryoablation model [31]. Initial results reported a statistically significant improvement in patient understanding $(p=0.007)$ from explanation of cryoablation with $3 \mathrm{DMs}$ compared to $2 \mathrm{D}$ imaging but, after adjusting for the physician providing the education, the 3DM didn't show a significant improvement anymore $(p=0.22)$.

\section{Surgical Training and 3DP}

Recent papers have underlined how urology trainees are less prone to participate during surgeries in the operating room due to more complex and minimally invasive procedures being introduced [32]. A lower exposure of residents to major procedures, as well as for novice inexperienced surgeons, is leading to poor satisfaction of surgical training and lower confidence performing surgeries independently [33]. Moreover, COVID-19 pandemic has rapidly affected surgical training due to the substantial decrease in election procedures in favor of urgent cases [34-37]. In this scenario, surgical training in urology may suffer more than before, and pursuing innovation in learning surgeries needs to be a cornerstone of trainees education that, with new tools, could even be implemented [38]. Considering this background, another possible application of $3 \mathrm{DMs}$ is simulation-based training (hands-on surgical practice) for novice and inexperienced surgeons. 3DMs could provide a safe scenario for training, especially for residents, without harming patients and guarantying always the standard of care. Monda et al. recently evaluated 3D printed molds of a patient's kidney with renal mass as a training tool for robotic NSS [39]. Twenty-four surgeons of different training levels performed four trials simulations for each one on silicone renal tumor models. A dedicated questionnaire regarding the realism and the overall feeling of the model, and usefulness for surgical training, was administered, and overall results were, respectively, 79.2 and 90.2. Renal artery clamping times, preserved renal parenchyma, positive margins, and Global Evaluative Assessment of Robotic Skills (GEARS) scores were all found to improve $(p<$ $0.001, p=0.025, p=0.024, p \leq 0.020, p \leq 0.006$, respectively) even if clamping times and GEARS scores proved to be significantly better in experienced surgeons hands ( $p \leq$ $0.005, p \leq 0.025$, respectively).

Ghazi et al., in a prospective study, created a simulated inanimate model made of poly-vinyl alcohol (PVA) hydrogels using a patient's CT scan with a 42-mm upper pole renal tumor (R.E.N.A.L. score 7) and stiffened to the desired consistency in order to simulate live surgery [40]. To replicate the entire surgical procedure, the kidney replica was layered in its anatomical configuration and surrounded by perinephric fat, neighboring organs, and posterior abdominal musculature. All steps of RAPN were simulated. The model resulted to have good face and content validity (average score of $3 / 5$ and $4 / 5$, respectively), providing a useful tool for evaluating and even improving surgical skills. Statistically significant difference was demonstrated in operative time (OT), ischemia time (IT), surgical margins, and EBL (all values had $p<0.01$ ). During the same year, 3D printed renal models with enhancing masses were tested on 23 first-year medical trainee for characterization, localization, and understanding of renal malignancy [41]. The 6 renal models were printed from a transparent plastic resin, and the tumor was delineated by a red hue. To medical trainee were asked to complete R.E.N.A.L. nephrometry score separately using 2D images and 3DMs and then complete a questionnaire about the experience. Overall R.E.N.A.L. score accuracy was significantly improved with the $3 \mathrm{DM}(p<0.01)$. In particular, $\mathrm{R} \mathrm{N}$ and $\mathrm{L}$ components of the score (radius, nearness and location) showed the higher improvement $(p<0.001)$ using the models. All these findings suggest that 3DP could help to improve trainees' understanding and characterization of renal masses. Furthermore, when compared to expert urologists, the interrater agreement improved with the 3DMs ( $p=$ 0.002). Marconi et al. showed how 3DMs of 15 patients scheduled for laparoscopic nephrectomy (LN) helped in identifying anatomic structures more quickly and accurately [42]. The lower the experience the higher the improvement, so medical students had the highest benefit $(53.9 \% \pm$ $4.14 \%$ of correct answers with 3DMs), instead of experienced urologists and radiologists. Moreover, time was almost $50 \%$ shorter than reviewing 2D CT scans $(60.67 \pm$ $25.5 \mathrm{~s}$ vs $127.04 \pm 35.91 \mathrm{~s}$, respectively).

About complex renal calculi, few authors have explored the possibility of surgical training using 3DMs. Firstly, in 2008, a French group exploiting a rapid prototyping technique created patient-specific silicone $3 \mathrm{DMs}$ from $\mathrm{CT}$ scans allowing surgical team and residents to train on the model before surgery, predicting difficulties due to patient's anatomy [43]. After training, the patient underwent PCNL without complications and discharged at postoperative day 1. On the other hand, only one patient has been enrolled, and few surgical outcomes were evaluated. Subsequently, Stone et al. evaluated 15 consecutive PCNL 
performed by a single urologist [44]. Among these, 7 patients had specific 3DMs used for preoperative rehearsal and training. In addition to patients' collecting system and staghorn calculi, also the kidney, spine, and posterior abdominal wall were created and assembled. All steps of a PCNL were simulated, including fluoroscopic access. Outcomes from the first 8 patients without prior rehearsal were compared to the $3 \mathrm{D}$ group, showing that mean fluoroscopy time was significantly lower in the second group (6.2 and $12.7 \mathrm{~min}$, respectively, $p=0.03$ ), but the higher improvement was registered on the average number of percutaneous needle access attempts that resulted to be lower in the 3D group ( 1.8 vs 5 attempts, $p<0.001)$. Antonelli et al. have gone beyond the scope of surgical training studying the benefits of a novel device (polyethylene sack called "PercSac") deployed into a 3D printed collecting system to capture stones and their fragments during PCNL simulations, in order to prevent stone migration [45]. The average time for stone fragmentation resulted to be significantly shorter in the PercSac group (217 s vs $340 \mathrm{~s}$ of the control group, $p=0.028$ ), and total time for complete stone was significantly shorter too (293 s vs 376 $\mathrm{s}, p=0.047)$. In vitro simulation provided a safe environment for training and testing the efficacy of the novel device, laying the groundwork for in vivo surgeries.

Renal access is one of the most important and complex steps in learning PCNL and ideally should be practiced outside the operating room particularly for residents. Simulations could be expensive and time-consuming. To satisfy the need of a cheap but accurate 3DM for PCNL training, Turney et al. successfully produced water-soluble plastic 3DMs of human collecting system to safely practice the fluoroscopy triangulation for percutaneous renal puncture [46]. However, results focused on costs while no estimation of number of punctures needed was reported neither improvement of surgical skills. The reduction of caseload and the increasing focus on patient safety have impacted on resident surgical training. Ghazi and colleagues validated a full-immersion platform for simulation before PCNL [47]. After producing 3D human pelvicalyceal system, kidney and adjacent structures, all steps of PCNL (percutaneous renal access, nephroscopy, and lithotripsy) were simulated by 5 experts and 10 novices from both international radiology (access only) and urology (full procedure) departments. 3DMs were rated high on realism and educational effectiveness and provided a useful tool for surgical simulation and training and also for skill evaluation before hands-on procedure. The greatest impact resulted on teaching and refining technical skills (4.71/5) as well as evaluation of performance (4.57/5). Obviously, it was registered a significant difference between experts and novices in mean fluoroscopy time, number of percutaneous access attempts, and needle repositioning.

\section{Augmented Reality (AR)}

AR refers to the alignment or superimposition of intraoperative, or, more commonly, preoperative imaging onto a patient's actual images or video, in real time. This allows the surgeon to simultaneously assimilate important visual information from the operative field with imaging modalities that usually play a passive role within the operating room (US, CT, MRI). The reconstructed images are registered onto anatomic landmarks and tracked by the computer according to the surgeon's tissue manipulation and the camera movements.

The 3D virtual models (3DVM) have been increasingly utilized in a virtual environment for medical education and surgical planning over the last decade to provide an increased understanding of kidney anatomy.

Head-mounted display systems have been proposed during preoperative planning to visualize 3DMs as holograms. A mixed-reality tool using zSpace workstation (a computer connected to a stereoscopic screen which allows to visualize virtual objects) was developed by Antonelli and colleagues [48]. A simulation environment can be visualized on the real one, and this experience seemed to improve preoperative planning for partial nephrectomy. Compared with a CT scan, mixedreality technology could provide much detailed anatomical information. Augmented reality, properly linked with operating systems, allows to add information to the real environment and to overlay $3 \mathrm{D}$ constructed virtual images. Nowadays, it is possible to visualize kidney $3 \mathrm{D}$ reconstructions as holograms in a mixed-reality environment. Porpiglia et al. pioneering study showed that augmented reality is a feasible and useful technology in an intraoperative setting [49]. Hyper accuracy 3D (HA3D) models were integrated with the Da Vinci robot and used during partial nephrectomies for selective clamping. This augmented reality experienced resulted to be as valid as the cognitive guidance with the addition that the surgeon could stay constantly focused on the operative field. The excision phase of $\mathrm{PN}$ can almost surely be considered the hardest step and an additional guidance by augmented reality showed promising results.

A similar study was conducted by Checcucci et al during a urological international meeting organized at their Institution on January 2019 [50]. Surgeons' perception of mixed reality for PN was evaluated. HA3D were performed based on preoperative CT scans. Then, a virtual environment was created with the possibility to interact with 3DMs by using HoloLens. This mixed reality setting scored very high on both surgical planning (8/10) and anatomical accuracy $(9 / 10)$. Furthermore, participants enthusiastic about its potential role in understanding of surgical complexity: after HoloLens mixed reality experience, 64.4 and $44.4 \%$ of the participants would have change their clamping and/or resection approach.

Singla et al. augmented-reality system provided an instrument tracking for excision phase [51]. Surgeries were 
successfully performed, and, by this tracking system, the amount of health parenchyma excised was significantly reduced (from $30.6 \pm 5.5$ to $17.5 \pm 2.4 \mathrm{ml}, p<0.05$ ) as well as the difference depth from the tumor underside to cut resulted to be statistically significant (from $10.2 \pm 4.1$ to $3.3 \pm 2.3 \mathrm{~mm}$, $p<0.05$ ).

Recently, a system which allows to overlap endoscopic images on 3DVM was develop and experienced during RAPN. Kobayashi et al. used this tool and evaluated the skills of two expert surgeons on identification and dissection of the renal artery [52]. This technology showed how the number of inefficient robotic motions was significantly reduced. A single center experience on preoperative patient counseling was reported by Wake et al. [26]. A 5-point Likert scale was used to evaluate overall comprehension of clinical cases after mixedreality experience using HoloLens to visualize 3DMs. Compared with mixed reality, 3DMs showed better results in the understanding of clinical cases.

Even if NSS is the most frequent surgery in which AR has been applied, endoscopic surgery, specifically for complex renal stones, has been tested with this immersive new tool. From 2017 to 2018, Parkhomenko et al. evaluated four surgeons of different expertise in PCNL used immersive virtual reality (iVR) models during preoperative planning [53]. The new technology improved surgeon's understanding of the optimal calix entry and stone location and conformation $(p<$ 0.01) than CT imaging alone, altering the operative approach in $40 \%$ of cases. In patients that tried iVR, an important reduction of preoperative anxiety due to an improved comprehension of surgery was registered. The retrospective matchedpaired analysis showed how iVR group had a statistically significant decrease in EBL (50 vs $100 \mathrm{~mL}, p<0.01$ ), fluoroscopy time (180 vs $226 \mathrm{~s}, p<0.01)$, as well as a fewer punctures (1.13 vs $1.46, p=0.09)$ and a higher SFR (39\% vs $20 \%, p=0.15$ ).

Similarly, a Turkish group evaluated a novel software to calculate the correct access point and angle for PCNL by using pre-operative CT [54]. Two scans, $27 \mathrm{~s}$ and $10 \mathrm{~min}$ after injection of contrast agent, were taken in prone PCNL position. In an augmented reality setting, 3DM was placed virtually onto real object and then calculated access point in 50 patients. According to the calculated direction angle, an access needle was displayed virtually on the object. Accuracy of insertion of needle was checked by feeling crepitation on stone surface and observing tip of needle touching stone in a control CT scan. However, the authors stated that further research is required to test its accuracy and safety in humans.

\section{Conclusion}

Several applications of 3DP have been proposed in the last few years in many fields. As far as innovation in 3DP technology gets better, 3D patient-specific models are becoming more affordable and widespread, even in smaller centers. Possible applications of 3DP in kidney surgery include surgical planning, patient education, training, and intraoperative AR, leading to goals never thought before. The disposability of 3D models in healthcare scenarios might improve surgical outcomes, learning curves of novice surgeons and residents, as well as patients' understanding and compliance, allowing a more shared surgical decision-making. Further studies aimed to standardize this technology application are needed to guarantee a new and universally shared way to approach kidney procedures.

\section{Declarations}

Conflict of Interest Francesco Esperto, Francesco Prata, Ana María Autrán-Gómez, Juan Gomez Rivas, Moises Socarras, Michele Marchioni, Simone Albisinni, Rita Cataldo, Roberto Mario Scarpa and Rocco Papalia each declare no potential conflicts of interest.

Human and Animal Rights and Informed Consent This article does not contain any studies with human or animal subjects performed by any of the authors.

\section{References}

1. Rankin TM, Giovinco NA, Cucher DJ, Watts G, Hurwitz B, Armstrong DG. Three-dimensional printing surgical instruments: are we there yet? J Surg Res. 2014;189:193-7.

2. Seol YJ, Kang HW, Lee SJ, Atala A, Yoo JJ. Bioprinting technology and its applications. Eur J Cardiothorac Surg. 2014;46:342-8.

3. Cacciamani GE, Okhunov Z, Meneses AD, Rodriguez-Socarras ME, Rivas JG, Porpiglia F, et al. Impact of three-dimensional printing in urology: state of the art and future perspectives. a systematic review by ESUT-YAUWP group. Eur Urol. 2019;76:209-21.

4. Van Poppel H, Da Pozzo L, Albrecht W, et al. A prospective, randomised EORTC intergroup phase 3 study comparing the oncologic outcome of elective nephron-sparing surgery and radical nephrectomy for low-stage renal cell carcinoma. Eur Urol. 2011;59: 543-52.

5. Sun M, Trinh Q-D, Bianchi M, Hansen J, Hanna N, Abdollah F, et al. A non-cancer-related survival benefit is associated with partial nephrectomy. Eur Urol. 2012;61:725-31.

6. Reddy UD, Pillai R, Parker RA, Weston J, Burgess NA, Ho ET, et al. Prediction of complications after partial nephrectomy by RENAL nephrometry score. Ann R Coll Surg Engl. 2014;96(6): 475-9. https://doi.org/10.1308/003588414X13946184903522.

7. Silberstein JL, Maddox MM, Dorsey P, Feibus A, Thomas R, Lee BR. Physical models of renal malignancies using standard crosssectional imaging and 3-dimensional printers: a pilot study. Urology. 2014;84:268-72. https://doi.org/10.1016/j.urology. 2014.03.042.

8. Zhang Y, Ge HW, Li NC, Yu CF, Guo HF, Jin SH, et al. Evaluation of three-dimensional printing for laparoscopic partial nephrectomy of renal tumors: a preliminary report. World J Urol. 2016;34:533-7.

9. Wake N, Rude T, Kang SK, Stifelman MD, Borin JF, Sodickson $\mathrm{DK}$, et al. 3D printed renal cancer models derived from MRI data: 
application in pre-surgical planning. Abdom Radiol (NY). 2017;42:1501-9.

10. Porpiglia F, Bertolo R, Checcucci E, et al. Development and validation of 3D printed virtual models for robot-assisted radical prostatectomy and partialnephrectomy: urologists' and patients' perception. World J Urol. 2018;36:201-7.

11. von Rundstedt FC, Scovell JM, Agrawal S, Zaneveld J, Link RE. Utility of patient-specific silicone renal models for planning and rehearsal of complex tumour resections prior to robot-assisted laparoscopic partial nephrectomy. BJU Int. 2017;119:598-604.

12. Maddox MM, Feibus A, Liu J, Wang J, Thomas R, Silberstein JL. 3D-printed soft-tissue physical models of renal malignancies for individualized surgical simulation: a feasibility study. J Robot Surg. 2018;12:27-33.

13. Kyung YS, Kim N, Jeong IG, Hong JH, Kim CS. Application of 3D printed kidney model in partial nephrectomy for predicting surgical outcomes: a feasibility study. Clin Genitourin Cancer. 2019;17:e878-e84. https://doi.org/10.1016/j.clgc.2019.05.024.

14. Fan G, Meng Y, Zhu S, Mingji Y, Mingfeng L, Feiping L, et al. Three-dimensional printing for laparoscopic partial nephrectomy in patients with renal tumours. J Int Med Res. 2019;47:4324-32. https://doi.org/10.1177/0300060519862058.

15. Komai Y, Sugimoto M, Gotohda N, Matsubara N, Kobayashi T, Sakai Y, et al. Patient-specific 3-dimensional printed kidney designed for "4D" surgical navigation: a novel aid to facilitate minimally invasive off-clamp partial nephrectomy in complex tumor cases. Urology. 2016;91:226-33.

16. Khan SR, Pearle MS, Robertson WG, Gambaro G, Canales BK, Doizi S, et al. Kidney stones. Nat Rev Dis Primers. 2016;2:16008. https://doi.org/10.1038/nrdp.2016.8.

17. Türk C, Petř́k A, Sarica K, Seitz C, Skolarikos A, Straub M, et al. EAU Guidelines on interventional treatment for urolithiasis. Eur Urol. 2016;69(3):475-82. https://doi.org/10.1016/j.eururo.2015. 07.041 Epub 2015 Sep 4.

18. de Souza Melo PA, Vicentini FC, Beraldi AA, Hisano M, Murta $\mathrm{CB}$, de Almeida Claro JF. Outcomes of more than 1000 percutaneous nephrolithotomies and validation of Guy's stone score. BJU Int. 2018;121(4):640-6. https://doi.org/10.1111/bju.14129 Epub 2018 Feb 1 .

19. Gadzhiev N, Brovkin S, Grigoryev V, Tagirov N, Korol V, Petrov S. Sculpturing in urology, or how to make percutaneous nephrolithotomy easier. J Endourol. 2015;29(5):512-7. https://doi. org/10.1089/end.2014.0656.

20. Xu Y, Yuan Y, Cai Y, Li X, Wan S, Xu G. Use 3D printing technology to enhance stone free rate in single tract percutaneous nephrolithotomy for the treatment of staghorn stones. Urolithiasis. 2020;48(6):509-16. https://doi.org/10.1007/s00240-019-01164-8.

21. Bianchi L, Schiavina R, Barbaresi U, Angiolini A, Pultrone CV, Manferrari F, et al. 3D Reconstruction and physical renal model to improve percutaneous puncture during PNL. Int Braz J Urol. 2019;45(6):1281-2. https://doi.org/10.1590/S1677-5538.IBJU. 2018.0799.

22. Brehmer M, Beckman MO, Magnusson A. Three-dimensional computed tomography planning improves percutaneous stone surgery. Scand J Urol. 2014;48(3):316-23. https://doi.org/10.3109/ 21681805.2013.876552 Epub 2014 Feb 12.

23. Li H, Chen Y, Liu C, Li B, Xu K, Bao S. Construction of a threedimensional model of renal stones: comprehensive planning for percutaneous nephrolithotomy and assistance in surgery. World J Urol. 2013;31:1587-92.

24. Manning TG, O'Brien JS, Christidis D, et al. Three dimensional models in uro-oncology: a future built with additive fabrication. World J Urol. 2018;36:557-63.

25. Canter D, Kutikov A, Manley B, et al. Utility of the R.E.N.A.L. nephrometry scoring system in objectifying treatment decision- making of the enhancing renal mass. Urology. 2011;78:1089-94. https://doi.org/10.1016/j.urology.2011.04.035.

26. Wake N, Rosenkrantz AB, Huang R, Park KU, Wysock JS, Samir $\mathrm{S}$, et al. Patient-specific 3D printed and augmented reality kidney and prostate cancer models: impact on patient education. 3D Print Med. 2019;5:4. https://doi.org/10.1186/s41205-019-0041-3.

27. Teishima J, Takayama Y, Iwaguro S, Hayashi T, Inoue S, Hiedka $\mathrm{K}$, et al. Usefulness of personalised three-dimensional printed model on the satisfaction of preoperative education for patients undergoing robot-assisted partial nephrectomy and their families. Int Urol Nephrol. 2018;50:1061-6. https://doi.org/10.1007/ s11255-018-1881-2.

28. Zhang Y, Ge HW, Li NC, Yu CF, Guo HF, Jin SH, et al. Evaluation of three-dimensional printing for laparoscopic nephrectomy of renal tuours: a preliminary report. World J Urol. 2016;34:533-7. https:// doi.org/10.1007/s00345-015-1530-7.

29. Bernhard JC, Isotani S, Matsugasumi T, Duddalwar V, Hung AJ, Suer E, et al. Personalized 3D printed model of kidney and tumor anatomy: a useful tool for patient education. World J Urol. 2016;34: 337-45. https://doi.org/10.1007/s00345-015-1632-2.

30. Atalay HA, Canat HL, Ulker V, Alkan I, Ozkuvanci U, Altunrende F. Impact of personalized three-dimensional (3D) printed pelvicalyceal system models on patient information in percutaneous nephrolithotripsy surgery: a pilot study. Int Braz J Urol. 2017;43: 470-5. https://doi.org/10.1590/s1677-5538.ibju.2016.0441.

31. Schmit C, Matsumoto J, Yost K, Alexander A, Ness L, Kurup AN, et al. Impact of a 3D printed model on patients' understanding of renal cryoablation: a prospective pilot study. Abdom Radiol (NY). 2019;44:304-209. https://doi.org/10.1007/s00261-018-1710-1.

32. Carrion DM, Rodriguez-Socarrás ME, Mantica G, Esperto F, Cebulla A, Duijvesz D, et al. Current status of urology surgical training in Europe: an ESRU-ESU-ESUT collaborative study. World J Urol. 2020;38(1):239-46. https://doi.org/10.1007/ s00345-019-02763-1.

33. Carrion DM, Gómez Rivas J, Esperto F, Patruno G, Vasquez JL. Current status of urological training in Europe. Arch Esp Urol. 2018;71(1):11-7 English.

34. Pang KH, Carrion DM, Rivas JG, Mantica G, Mattigk A, Pradere B. Esperto F; European society of residents in urology. The impact of COVID-19 on European Health care and urology trainees. Eur Urol. 2020;78(1):6-8. https://doi.org/10.1016/j.eururo.2020. 04.042 .

35. Amparore D, Claps F, Cacciamani GE, Esperto F, Fiori C, Liguori $\mathrm{G}$, et al. Impact of the COVID-19 pandemic on urology residency training in Italy. Minerva Urol Nefrol. 2020;72(4):505-9. https:// doi.org/10.23736/S0393-2249.20.03868-0.

36. Esperto F, Papalia R, Pang KH, Cataldo R, Scarpa RM. What is the role of residents during a pandemic? Minerva Urol Nefrol. 2020;72(3):387-8. https://doi.org/10.23736/S0393-2249.20. 03903-X.

37. Esperto F, Prata F, Civitella A, Pang KH, Marchioni M, Tuzzolo P, et al. Implementation and strategies to ensure adequate coordination within a urology department during the COVID-19 pandemic. Int Braz J Urol. 2020;46(suppl.1):170-80. https://doi.org/10.1590/ S1677-5538.IBJU.2020.S122.

38. Claps F, Amparore D, Esperto F, Cacciamani G, Fiori C, Minervini A, et al. Smart learning for urology residents during the COVID-19 pandemic and beyond: insights from a nationwide survey in Italy. Minerva Urol Nefrol. 2020;72(6):647-9. https://doi.org/10.23736/ S0393-2249.20.03921-1.

39. Monda SM, Weese JR, Anderson BG, Vetter JM, Venkatesh R, $\mathrm{du} \mathrm{K}$, et al. Development and validity of a silicone renal tumor model for robotic partial nephrectomy training. Urology. 2018;114:114-20. 
40. Ghazi A, Stone J, Candela B, et al. Simulated inanimate model for physical learning experience (simple) for robotic partial nephrectomy using a 3d printed kidney model. J Urol. 2015;193:e778.

41. Knoedler M, Feibus AH, Lange A, Maddox MM, Ledet E, Thomas $\mathrm{R}$, et al. Individualized physical 3-dimensional kidney tumor models constructed from 3-dimensional printers result in improved trainee anatomic understanding. Urology. 2015;85:1257-61.

42. Marconi L, Desai MM, Ficarra V, Porpiglia F, van Poppel H. Renal preservation and partial nephrectomy: patient and surgical factors. Eur Urol Focus. 2016;2:589-600.

43. Bruyère F, Leroux C, Brunereau L, Lermusiaux P. Rapid prototyping model for percutaneous nephrolithotomy training. $\mathrm{J}$ Endourol. 2008;2(1):91-6.

44. Stone J, Bonamico R, Erturk E, Ghazi A. PD41-01 improved surgical outcomes after preoperative rehearsal using $3 \mathrm{D}$ printed patient specific simulation for percutaneous nephrolithotomy (PCNL). J Urol. 2017;197(4):e807.

45. Antonelli JA, Beardsley H, Faddegon S, Morgan MSC, Gahan JC, Pearle MS, et al. A novel device to prevent stone fragment migration during percutaneous lithotripsy: results from an in vitro kidney model. J Endourol. 2016;30(11):1239-43.

46. Turney BW. A new model with an anatomically accurate human renal collecting system for training in fluoroscopy-guided percutaneous nephrolithotomy access. J Endourol. 2014;28(3):360-3. https://doi.org/10.1089/end.2013.0616.

47. Ghazi A, Campbell T, Melnyk R, Feng C, Andrusco A, Stone J, et al. Validation of a full-immersion simulation platform for percutaneous nephrolithotomy using three-dimensional printing technology. J Endourol. 2017;31(12):1314-20. https://doi.org/10.1089/ end.2017.0366.

48. Antonelli A, Veccia A, Palumbo C, Peroni A, Mirabella G, Cozzoli A, et al. Holographic reconstructions for preoperative planning before partial nephrectomy: a head-to-head comparison with standard CT scan. Urol Int. 2019;102:212-7.

49. Porpiglia F, Fiori C, Checcucci E, Amparore D, Bertolo R. Hyperaccuracy three-dimensional reconstruction is able to maximize the efficacy of selective clamping during robot-assisted partial nephrectomy for complex renal masses. Eur Urol. 2018;74:651-60.

50. Checcucci E, Amparore D, Pecoraro A, Peretti D, Aimar R, De Cillis $\mathrm{S}$, et al. 3D mixed reality holograms for preoperative surgical planning of nephron-sparing surgery: evaluation of surgeons' perception. Minerva Urol Nefrol. 2019. https://doi.org/10.23736/ S0393-2249.19.03610-5.

51. Singla R, Edgcumbe P, Pratt P, Nguan C, Rohling R. Intraoperative ultrasound-based augmented reality guidance for laparoscopic surgery. Health Technol Lett. 2017;4:204-9.

52. Kobayashi S, Cho B, Huaulme' A, et al. Assessment of surgical skills by using surgical navigation in robot-assisted partial nephrectomy. Int J Comput Assist Radiol Surg. 2019;14:1449-59.

53. Parkhomenko E, O'Leary M, Safiullah S, Walia S, Owyong M, Lin $\mathrm{C}$, et al. Pilot assessment of immersive virtual reality renal models as an educational and preoperative planning tool for percutaneous nephrolithotomy. J Endourol. 2019;33(4):283-8. https://doi.org/10. 1089/end.2018.0626.

54. Akand M, Civcik L, Buyukaslan A, Altintas E, Kocer E, Koplay $\mathrm{M}$, et al. Feasibility of a novel technique using 3-dimensional modeling and augmented reality for access during percutaneous nephrolithotomy in two different ex-vivo models. Int Urol Nephrol. 2019;51(1):17-25. https://doi.org/10.1007/s11255018-2037-0.

Publisher's Note Springer Nature remains neutral with regard to jurisdictional claims in published maps and institutional affiliations. 